\title{
An "outside-in" processing strategy in the perception of form
}

\author{
BRUCE EARHARD and HEATHER WALKER \\ Dalhousie University, Halifax, Nova Scotia, Canada
}

\begin{abstract}
The discriminability of line segments in a number of different geometric forms was examined. The procedure used was to have one of the lines within a given form drawn thinner than the other lines. The location of the thin line varied randomly within the form, and subjects were required to detect its location. Three observations were made. First, outer line elements were discriminated more accurately than inner line elements. Second, the outer-line advantage appeared to reflect an attentional strategy: By presenting inner and outer lines independently, and informing subjects in advance as to whether target lines would be in the inner or outer area of the form, the outer line advantage could be eliminated. Third, consonant with a global attentional strategy, and in opposition to any retinally tied, peripheral-to-central scanning process, an outer-line advantage was evident regardless of whether the fixation point was positioned within or outside the forms, or whether outer lines were more peripheral than inner lines.
\end{abstract}

Both introspective analysis and experimentation tell us that not all facets of a perceived object or scene are registered simultaneously. Some aspects are immediately discerned; others are rendered up only after a more protracted inspection. The isolation and specification of factors that are responsible for, or influence, the course of scene or form analysis has become a major object of much contemporary research in cognitive psychology. Two general approaches have been taken.

One approach has concentrated on the characteristics of the stimulus configuration and the way in which they contribute to the discrimination of constituent elements of the form or scene. For example, Prinzmetal and Banks (1977) have examined the degree to which Gestalt-like contexts can impair or facilitate detection of constituent elements or components of a given form. Weisstein and her associates (e.g., Lanze, Weisstein, \& Harris, 1982; Weisstein \& Harris, 1974; Williams \& Weisstein, 1978) have attempted to demonstrate that coherence, connectedness, and three-dimensionality serve to enhance discriminability of constituent line elements in outline forms. In a variant of this approach, Pomerantz, Sager, and Stoever (1977) and Treisman and Paterson (1984) have endeavored to demonstrate how constituent components of form can combine with one another to form highly discriminable emergent features.

The other approach, with which the present paper is aligned, has been to try to delineate the character of general processing dispositions, that is, commonly used strategies and heuristics involved in the processing of visual scenes or forms. Representative of this approach

This research was supported by a grant from the Natural Science Research and Engineering Council of Canada. Address correspondence to B. Earhard, Department of Psychology, Dalhousie University, Halifax, Nova Scotia, Canada B3H 4JI. is the work of Navon (1977) on global-local processing dispositions. His work suggests that global structural features are available within the perceptual system before local detail, and that such global information provides a basis for directing the search for local detail. Although there are constraints on the generality of the global-tolocal analytic operations (Kinchla \& Wolfe, 1979; Pomerantz, 1983), Navon has assembled a substantial base of support for this heuristic (Navon, 1981a, 1981b; Navon \& Norman, 1983).

It seems reasonable to assume that there are a variety of other processing dispositions. One often cited possibility is that the analytic process begins at the outer boundaries and proceeds inward. Empirical evidence for the general deployment of such a processing disposition in the perception of global, geometric-type forms is wanting. In discussing this heuristic, the standard reference is to Waltz's (1975) model of scene analysis, but his account is theoretical, rather than empirical. Some authors (e.g., McClelland \& Miller, 1979) have suggested that aspects of their data are most readily accounted for by an "outside-in" processing strategy, and others (e.g., Lanze et al., 1982) cite unpublished data to support arguments favoring outside-in processing of geometric forms. At present, the strongest published evidence favoring such a processing disposition remains experimentation on unidimensional letter strings. It has been found that if letters are presented simultaneously in both visual fields, outer letters are discriminated more readily than immediately adjacent inner letter elements (e.g., White, 1976; Wolford \& Hollingsworth, 1974). It has also been found that if pairs of letters are presented separately in the left or right visual fields, the peripheral letters are discriminated more accurately than the more central letters (e.g., Banks, Larson, \& Prinzmetal, 1979; Banks \& White, 1984). 
Although investigations of lateral asymmetries with alphabetic materials show that peripheral letters seem to suffer less interference than more centrally located letters, there is reason to be very cautious about assuming that local interference effects demonstrated with isolated letter clusters will generalize directly to more global geometric forms. Available evidence suggests that local interference effects often tend to be overshadowed when a more global structure is provided. This is readily illustrated by the object-line effect (Williams \& Weisstein, 1978), which demonstrates that individual lines are discriminated more accurately when combined within the context of a global form than when presented alone. This is exactly opposite to what would be expected on the basis of lateral interference at the local level. As Williams and Weisstein (1978) point out, the proximity of adjacent lines should interfere with, rather than facilitate, discrimination of a target.

There are also reasons to expect the generalization to geometric forms to be less than perfect with respect to the role of other processes. For example, because there are obvious differences in the type of materials and presentation format used in the two types of study, it seems likely that attentional mechanisms play a more prominent part in the processing of more global geometric forms than has been suggested by a number of studies using alphabetic materials. In lateral asymmetry studies, letter strings are presented either across the field of vision or, more commonly, in either the left or right visual field. Inner and outer target elements requiring attention are thus either on the left or the right. In studies using geometric forms, on the other hand, a subject has to attend not only to inner and outer components on the left or the right, but also to inner and outer components in the upper and lower parts of the form. Given that attention has to be spread more broadly, it seems quite possible that the allocation of attention may well fall under voluntary control to a much greater degree than in unidimensional letter discrimination tasks. Consonant with this speculation is evidence that subjects can accommodate to imposed variations in report requirements much more readily when geometric forms are presented than when letter strings are presented (e.g., Bryden, 1960; Bryden, Dick, \& Mewhort, 1968).

To date, there has been no examination of the discriminability of the various line elements constituting an outline drawing, nor has there been any examination of the role played by attentional strategies in discriminating line detail. The principle reason such an examination has not taken place is that a technique for assessing these factors within geometric forms has not been available. ${ }^{1}$ The present study attempts to address this deficiency by using a technique that allows the examination of attentional processes and the discriminability of line detail in visual form without altering the overall character of the form. The technique involves making one line in a geometric form thinner than the other lines and requiring the subject to locate or detect the thin target line. By continuously changing the location of the thin line within the form, the discriminability of the components can be systematically assessed.

The first objective of the present study was to determine whether outer lines are more discriminable than interior lines in geometric forms. The second objective was to examine the degree to which voluntary attentional processes contributed to any apparent outer-line advantage when geometric forms were presented. The third objective was to discern if any observed outer-element advantage varied as a function of fixation-point location. The role of fixation point is important because if a difference between inner and outer lines were to be found only with a central, interior, fixation-point location, it would suggest a retinally tied, peripheral-to-foveal process, whereas finding the effect at other fixation-point locations would suggest a more general processing disposition.

\section{EXPERIMENT 1}

Experiment 1 was designed to provide preliminary information about the discriminability of line elements as a function of inner and outer locations within a conventional line drawing.

\section{Method}

Stimuli and Design. The study employed the two outline forms shown in Panel A of Figure 1. The two forms were presented independently, and subjects were required to discern whether all lines in the form presented were the same thickness or whether one line in the form was thinner than the other lines in the form. On half of the trials, all the lines were the same thickness; on the remaining trials, one of the nine line elements constituting each form was drawn thinner than the other lines composing the form. A thin line occurred in each of the nine possible locations equally often to ensure attention to the overall forms. The major source of interest was, however, in the discriminability of the four lines forming the diamond pattern $A B C D$, which, as Figure 1 shows, is an inherent part of both Form A and Form B. With the fixation point shown, all four lines are equidistant from one another and from the point of fixation. More important, the line elements $\mathrm{AB}$ and $\mathrm{BC}$ constitute outer line elements in Form $A$ and inner lines in Form B, whereas line elements $\mathrm{AD}$ and $\mathrm{DC}$ constitute inner line elements in Form A and outer line elements in Form B. Thus, one can look at the degree to which the same line elements are discerned when they constitute inner, as opposed to outer, line elements. To minimize the contribution of any variation in the drafting of individual stimulus forms, four stimulus presentation sets were prepared for both Form A and Form B. Each presentation set was composed of 10 versions of either Form A or Form B. In 9 of the 10 versions composing each set, one line element (a different one in each version) was shown thinner than the other line elements in the form. In the 10th version, all lines were drawn the same thickness. Thin lines were drawn with a $.25-\mathrm{mm}$ Koh-i-noor Rapidomatic pen, and regular lines with a .50 -mm pen. Forms subtended a visual angle of $.81^{\circ}$ horizontally, and $1.38^{\circ}$ vertically.

Procedure and Subjects. Stimulus Forms A and B were presented separately to all subjects. Half of the subjects began with Form A and the other half with Form B. Before stimulus presentations began, the subjects were presented with examples of all the 10 different versions of each form. They were told that the forms would be presented briefly, and that they were to say "yes" if they thought a thin line was evident and "no" if all lines were the same thickness. Additional instructions indicated that they could expect half the presentations in a given trial block to consist of forms contain- 


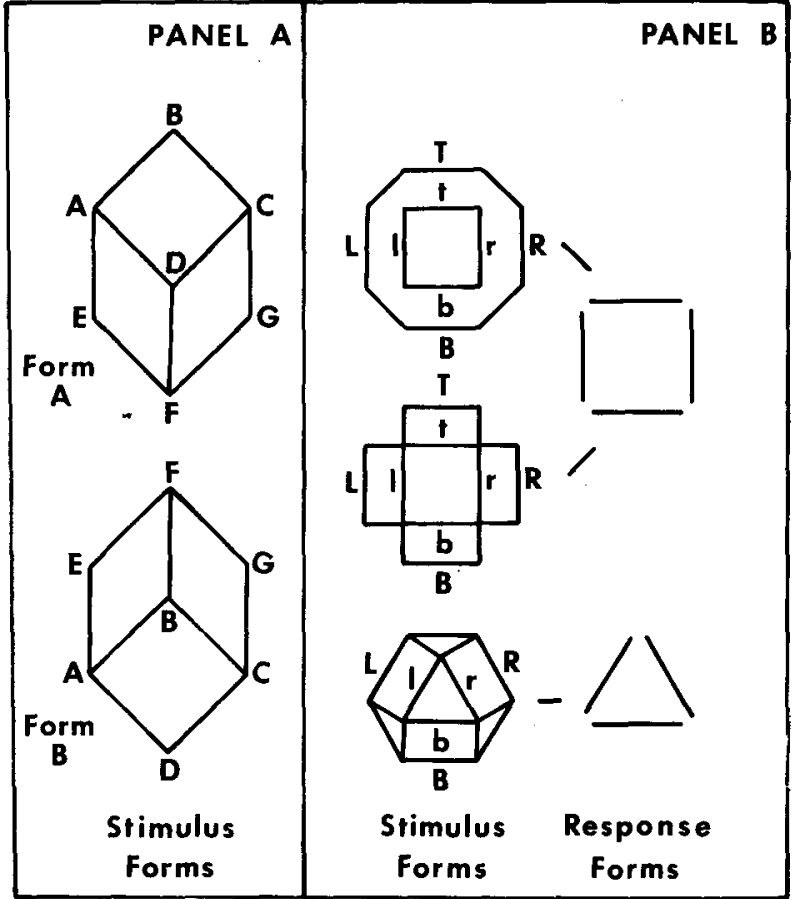

Figure 1. Panel A shows the forms used in Experiment 1. Fixationpoint location was at the center of the diamond form ABCD. In Panel B, the cross, octagon, and triangular-type stimulus forms used in Experiments 3 and 4 are shown on the left, and the response forms on the right. The fixation point was located at the center of each stimulus form during presentations. Upper- and lowercase letters are used to indicate paired inner and outer target lines. Letters are included to clarify exposition only, and were not part of the stimulus presentation.

ing a thin line. It was emphasized that each of the different line elements within the form would appear equally often in thin-line guise. It was stressed also that only one line in a form would be drawn thinner than the other lines, and that the task was only to detect the thin line, and not to specify its location. Stimulus forms were presented for a 50 -msec exposure by means of a Gerbrands two-field tachistoscope. The level of illumination of the exposure field was fixed at $5.63 \mathrm{~cd} / \mathrm{m}^{2}$, and the level of illumination of the preexposure field was adjusted for each subject to ensure an overall level of performance of $70 \%-80 \%$ correct responding for that subject. The mean level of illumination of the preexposure field was $2.13 \mathrm{~cd} / \mathrm{m}^{2}$ for Form A and $2.23 \mathrm{~cd} / \mathrm{m}^{2}$ for Form B. Two experimental sessions were required to complete the presentation of each form. Each session consisted of 2 18-item practice blocks followed by 12 18-item blocks of experimental presentations. Ten undergraduate students were recruited as subjects and were paid for their participation.

\section{Results}

The mean proportions of correct responses made to the three inner and six outer lines were calculated, and a clear outer-line advantage was evident. The proportion of correct outer-line discriminations was .75 and .73 for Forms $A$ and $B$, respectively, whereas the corresponding proportion of inner-line discriminations was .52 and .57 , respectively. An analysis of variance carried out on the aver- age proportion of correct responses made to outer and inner line elements showed a reliable difference between inner and outer line elements $[F(1,9)=12.71, p<.01]$. Although there was no overall difference in accuracy between the two forms [F $<1]$, a significant interaction was evident between inner and outer line elements and the two different forms employed $[F(1,9)=9.33, p<$ $.01]$. Because of the interaction, the two forms were analyzed independently. A reliable difference favoring outer over inner line elements was evident in the analysis performed on both Form $A$ and Form $B[F(1,9)=15.82$, $p<.01$, and $F(1,9)=9.05, p<.05$, respectively]. The interaction evident in the combined analysis reflects the fact that the magnitude of the difference favoring outer over inner lines is greater in Form A than in Form B.

The overall comparison of inner and outer lines, although suggestive, has a number of limitations. There are different numbers of inner and outer lines, and the lines compared vary in orientation and distance from the fixation point. A more meaningful analysis can be carried out on the diamond form $A B C D$, in which all line elements are equidistant from the fixation point and the same lines serve as inner structure in one form and outer structure in the other. When lines $A B$ and $B C$ served as outer lines and lines $A D$ and $D C$ as inner lines (Form $A$ ), lines $A B$ and $\mathrm{BC}$ were reported proportionally more accurately than lines $A D$ and $D C(.82$ vs. .63). When lines $A B$ and $B C$ served as inner lines, and $A D$ and $D C$ as outer lines (Form $\mathrm{B}$ ), lines $\mathrm{AB}$ and $\mathrm{BC}$ were reported proportionally less accurately than lines $A D$ and $D C$ (.63 vs. .76). An analysis of variance comparing the number of correct specifications of thin lines in positions $A B$ and $B C$ and in positions $A D$ and $D C$ in the two forms confirmed that detectability of target lines depended on whether targetline locations were inner or outer components of the form $[F(1,9)=6.32, p<.05]$. No other main effects or interactions in the analysis were reliable.

Experiment 1 demonstrates the utility of the detection procedure, and provides evidence confirming outer line elements are more discriminable than inner line elements.

\section{EXPERIMENT 2}

Experiment 2 was designed to test the generality of the outer-line advantage evident in Experiment 1 by ascertaining whether it would remain apparent when larger forms were used and a more natural response requirement was employed.

\section{Method}

Design. The size issue was approached by employing the two sets of forms shown in Figure 2. The forms in one set were similar in size to those used in the preceding experiment, and forms in the other set were twice as large. The more natural, holistic response issue was addressed by requiring subjects to base their responses on the entire stimulus form presented. After each presentation, subjects were required to indicate which of the four possible stimulus forms had been shown. As shown in Figure 2, stimulus forms were constructed by combining square and diamond shapes in such a way 


\section{Small Forms}
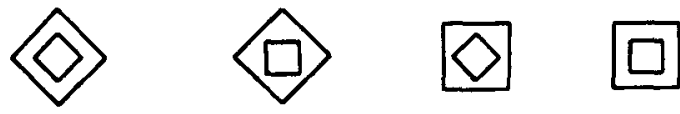

\section{Large Forms}
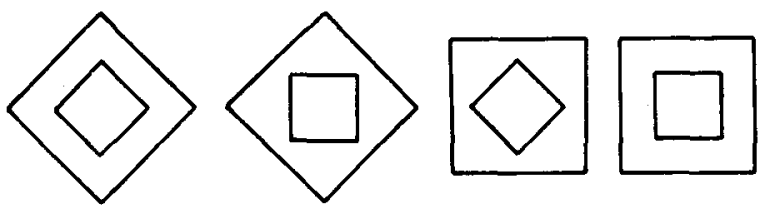

Figure 2. The sets of small and large forms used in Experiment 2. Fixation-point location was at the center of each form shown.

that each served equally often as inner and outer components in the set of stimulus forms used. Having the same shapes serve equally often as both inner and outer components of stimulus forms ensures that the two components are equally available and familiar to subjects, and allows any processing strategy, such as the use of partial information, to be applied equally to both the inner and outer components of target forms. By requiring subjects to attend to the entire form, and arranging stimulus parameters so as to ensure a performance level well above chance, but less than perfect, it is possible to determine whether inner and outer components are discerned with equal accuracy.

Stimuli. Individual forms were drawn on artist's board with a .25-mm Koh-i-noor Rapidograph pen. The construction of the forms was such that the outer components of the small forms were the same size as the inner components of the large forms. In the small forms, the inner square and diamond shapes subtended visual angles of $.57^{\circ}$ and $.81^{\circ}$, respectively, and the corresponding outer shapes subtended visual angles of $1.15^{\circ}$ and $1.62^{\circ}$, respectively. In the large forms, the visual angles subtended by the inner square and diamond shapes were $1.15^{\circ}$ and $1.62^{\circ}$, respectively, and the visual angles subtended by the outer shapes were $2.29^{\circ}$ and $3.24^{\circ}$, respectively.

Procedure and Subjects. Experimental sessions consisted of two practice blocks to familiarize subjects with the procedures, followed by six blocks of experimental presentations. There were 32 presentations in a block. Small and large forms were presented on alternate blocks and subjects were informed before each block whether the small or large forms were to be presented. A card on which the four different stimuli used in the experiment were drawn was continuously available to the subject. After each presentation, subjects were required to point to the stimulus form on the card to indicate the stimulus form presented. They were required to guess if in doubt. Stimulus forms were exposed for $20 \mathrm{msec}$. The level of illumination of the exposure field was held fixed at $2.16 \mathrm{~cd} / \mathrm{m}^{2}$, and the level of the preexposure field was adjusted to ensure an overall performance level of $50 \%-75 \%$ correct responding for each subject. The mean levels of the preexposure field illumination for the small and large forms were $3.09 \mathrm{~cd} / \mathrm{m}^{2}$ and $5.08 \mathrm{~cd} / \mathrm{m}^{2}$, respectively. Eight undergraduate students served as subjects in exchange for course credit points.

\section{Results}

The subjects correctly specified the exact form exposed on $61.7 \%$ of the presentations. Thus, the subjects were well above chance in their ability to specify both the inner and outer aspects of the forms presented. To separate out the difficulty the subjects experienced in dealing with inner, as opposed to outer, components of the form, accuracy on inner and outer components was analyzed independently. That is, if the subjects responded by selecting an alternative with the correct inner component, then that response was considered correct regardless of whether the outer component was correct. Similarly, if the subjects responded by selecting an alternative with the correct outer component, then that alternative was considered correct regardless of whether the inner component was correct. In the small-stimulus set, the outer form was reported correctly on $86.7 \%$ of the presentations, and the inner form on $68.2 \%$ of the presentations. A similar pattern was evident in the large-stimulus set. The outer form was designated correctly on $82.8 \%$ of the presentations and the inner form on $72.3 \%$ of the presentations. Thus, overall, the subjects were correct on $84.8 \%$ of the presentations with respect to the outer character of the form, but on only $70.3 \%$ of the presentations with respect to the inner character of the form. Analysis of variance showed this difference to be reliable $[F(1,7)=33.60$, $\mathrm{p}<.001]$. The advantage of outer over inner components is somewhat less pronounced for the larger forms, but the interaction was well short of significance $[F(1,7)=2.91$, $\mathrm{p}<.05]$. The data of Experiment 2 illustrate that even with a more holistic viewing and responding requirement and larger forms, outer components are reported more accurately than inner line components.

\section{EXPERIMENT 3}

Experiments 1 and 2 serve to confirm that outer line elements are reported more accurately than inner line elements when geometric forms are presented. The reason for this difference remains to be determined. It was pointed out in the introduction that there are reasons to suspect that attentional processes may assume a prominent role when geometric forms are used as stimuli. Before exploring the possibility that attentional factors are a major determinant of the outer-line advantage, it seemed reasonable to first determine whether the effect could be attributed to two simpler, sensory-based mechanisms.

The first possibility considered was that target-line elements might stand out and be more discriminable when part of the larger outer contour than when pressed in close proximity to one another as part of a smaller inner structural unit. That is, the advantage of outer over inner lines may not depend on whether they are outer or inner components of a given form, but rather on whether they constitute part of a larger or smaller structural unit. In forms such as those used in Experiments 1 and 2, the inner line components necessarily form a smaller structural unit than the outer line components. Navon and Norman (1983) have emphasized the importance of size, and suggest that larger perceptual components may be available earlier than smaller components. If relative size of inner and outer line structures is an instrumental factor in making target 
lines in the outer contours of a form more discriminable, then a target line should be more discriminable when part of a larger outer unit than when part of a smaller inner unit, even if the inner and outer structural components are presented separately.

The second possibility considered was that inner line elements may be interfered with more by the presence of outer contours than outer line elements are interfered with by the presence of inner line elements. Such a pattern has been observed in studies using alphabetic material (e.g., Wolford \& Hollingsworth, 1974).

Both of the above possibilities were considered by comparing discrimination of target lines under conditions in which the inner and outer components were presented separately and under conditions in which inner and outer units were combined to constitute a single form.

\section{Method}

Stimuli and Design. New stimulus forms were constructed. A procedure of pairing inner and outer line elements was followed in designing the forms, so as to ensure equality with respect to number, length, and orientation of inner and outer lines. The octagon and cross forms shown in Panel B of Figure 1 were used in the study. The subjects' task was to discern a thin target letter in the inner or outer parts of the form during a presentation, and to point immediately to the location of the line on the appropriate response form for that figure. As Figure 1 shows, a given pair of inner and outer lines having a similar location and orientation were represented by a single line on the response form. Thus, the same response was used to designate inner and outer locations for any given pair of target lines.

Target lines were located on the small inner component structure of a given form on half of the presentation blocks, and on the large outer component structure of the form on the remaining blocks. On half the presentations involving inner line targets, only the small inner line component structure was presented, and on the remaining presentations, the surrounding outer line structure was included. Similarly, on half of the presentations involving outer line structure, the large outer line structure was presented alone, and on the remaining presentations inner line components were included. This procedure was designed to assess the degree to which inner and outer line elements influenced discriminability of one another. To eliminate any voluntary tendency to allocate attention to outer parts of the forms presented, the subjects were informed prior to the start of each block whether targets would appear in the inner or outer components of the form. Individual forms were drawn in the same fashion as those employed in Experiment 1. The outer contours of the octagon and cross forms subtended visual angles of $1.15^{\circ}$; the inner square subtended $.57^{\circ}$

Procedure and Subjects. The subjects were provided with sample drawings showing the thin target line in each different inner and outer location for all versions of a given form. It was emphasized that they were to discern the location of the thin target letter and point to the location of the target line on the response form provided. The subjects were required to designate a location after each presentation, even if they were unsure of the correctness of their response. Two 32-item practice blocks were used to familiarize the subjects with the presentation procedure. Practice presentations were followed by six blocks of experimental presentations in each of two sessions.

Before each trial block, the subjects were told whether thin target lines would appear only in outer or inner locations. In addition, the subjects were informed before blocks involving inner targetline designations that the inner square would appear without an outer context on half of the presentations and with an outer context on the remaining presentations. Similarly, on trial blocks in which targets occupied outer contour locations, subjects were informed that the outer contour of the form would appear without an inner context on half of the presentations. Thin target lines occurred equally often in all four possible inner or outer positions. Presentation blocks of inner and outer target locations alternated with one another. Half the subjects started with inner target-line locations and half with outer target-line locations. A 50-msec exposure duration was used and the level of illumination of the exposure field was $5.63 \mathrm{~cd} / \mathrm{m}^{2}$. The level of illumination of the preexposure field was adjusted to ensure that overall accuracy of each subject was between $50 \%$ and $75 \%$ correct responding. The mean levels of illumination of the preexposure field for the cross and octagon forms were $6.04 \mathrm{~cd} / \mathrm{m}^{2}$ and $6.45 \mathrm{~cd} / \mathrm{m}^{2}$, respectively. To facilitate comparison of inner and outer line elements, adjustments in illumination of the preexposure field were made only after completion of both an inner and an outer presentation block. Two separate groups of 8 undergraduate subjects were presented with cross and octagon forms and received course credit for participating.

\section{Results}

Over 12 presentation blocks, a target line occupied each different inner and outer target line location in each of the four different variants of the cross and octagon form on 24 occasions. An analysis of variance was performed on the number of those 24 target lines that subjects correctly identified. A significant interaction reflecting a difference between the two forms when inner and outer lines were presented alone and when they were combined was evident $[\mathrm{F}(1,14)=5.47, \mathrm{p}<.05]$. Data from the two forms were therefore analyzed independently. Figure 3 shows the pattern of data obtained for the two forms. In spite of the interaction, the patterns of data obtained with the two forms were very similar. What is most obvious is that the relative difference in size of the inner and outer structural units does not contribute to the production of an outer-line advantage. Contrary to what would be expected according to this account, target lines were not more discriminable when part of the larger outer unit. The small differences evident, in fact, favored the inner component in both the cross and octagon forms, but these differences are not reliable $[\mathrm{F}(1,7)=4.97$, $\mathrm{p}<.05$, and $\mathrm{F}(1,7)=1.1, \mathrm{p}<.05$, respectively].

With respect to the second alternative, as expected, when inner and outer structural components were combined into a single unitary form, they interfered with one another. In both forms, discrimination of target lines was significantly more accurate when inner and outer components were presented separately $[\mathrm{F}(1,7)=75.16, \mathrm{p}<$ .001 , and $F(1,7)=206.37, p<.001$, respectively]. The crucial difference between the two forms producing the interaction in the overall analysis was in the character of the interference produced when inner and outer components were combined. In the cross form, inner lines were more impaired by the presence of outer lines than outer lines were impaired by the presence of inner lines $[F(1,7)=21.13, p<.01]$. In the octagon form, the asymmetry was much less apparent, and was not statistically reliable $[F(1,7)=3.76, p>.05]$. Although, in the cross form, inner line components suffered more by the addition of outer line components than outer line components suffered by the addition of inner line components, 

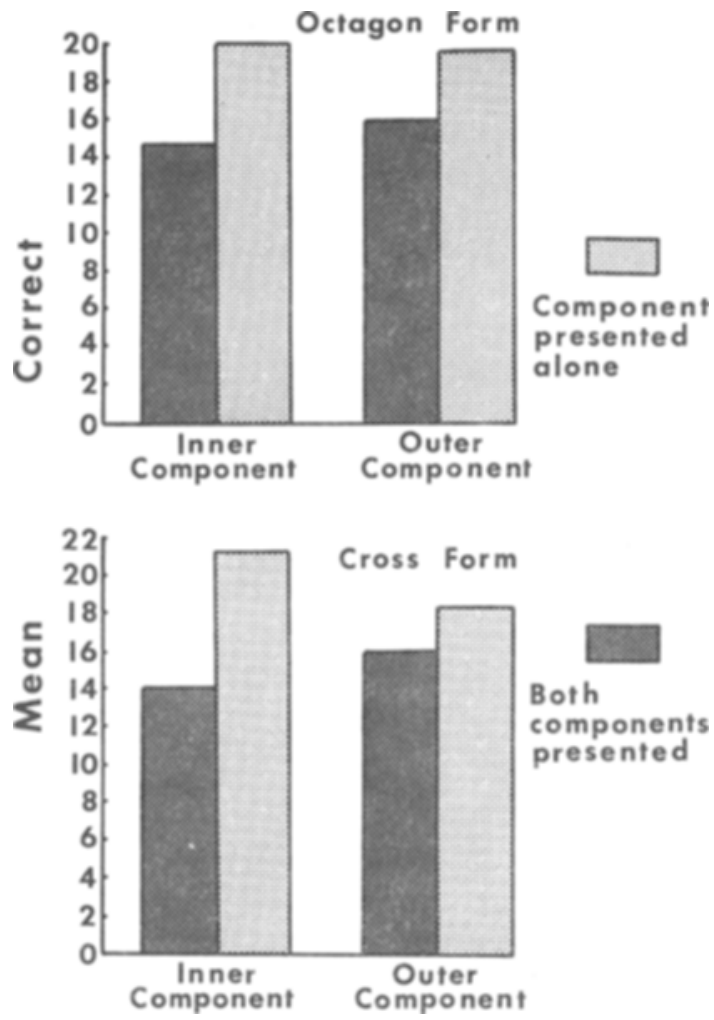

Figure 3. The mean level of accuracy with which inner and outer target line components were discriminated as a function of whether they were presented in isolation or as part of a cross or octagon form. The maximum number of correct responses possible in each condition was 24.

this was not sufficient to cause targets in the inner target lines to be discriminated less accurately than outer target lines in the composite forms. A Scheffé test showed that the difference in target discrimination was not reliable in either the cross or octagon forms at the .05 or .10 level of significance. Thus, the data obtained provide little support for the view that the outer-line advantage evident in Experiment 1 can be attributed either to greater size of the outer contour components or to asymmetrical interference.

The only remaining significant effect was that of the spatial position of the target line within the form, and this effect was apparent in the cross form $[F(3,21)=5.78$, $\mathrm{p}<.01]$, but not in the octagon form. Averaging over the 24 occasions each inner and outer target line was presented in each different stimulus configuration, the mean number of correct responses made to targets appearing on the top, right, bottom, and left positions of the cross form were $19.06,19.31,15.28$, and 16.28 , respectively. The subjects seemed to be equally effective in discerning targets on the top and on the right, and equally deficient in detecting target lines on the bottom and on the left. Application of the Scheffé test indicates that the difference between the top/right and the bottom/left positions is reliable $[F(3,21)=33.16, p<.05]$. The positional effects appear to be attributable to response bias. When the frequency with which different response positions were utilized was calculated for those trials on which errors were made, it was found that the mean number of erroneous responses made to the top, right, bottom, and left positions were $7.00,11.19,4.19$, and 3.78 , respectively. A Scheffé test showed that on error trials, as on target trials, subjects favored the top and right over the bottom and left positions in making their responses $[\mathrm{F}(3,21)=14.68$, $\mathrm{p}<.01]$.

\section{Discussion}

Experiment 2 sought to determine whether an advantage in discriminating outer line elements could be attributed simply to the greater size of the outer line elements, or to an asymmetry in interference. The data provided little support for either alternative. The differences in target-line discrimination were in the opposite direction to what would be expected if the size factor was involved. An asymmetry in interference was evident in only one of the two forms, and it was not sufficient to generate an outer-line advantage. The outer-line effect does not appear to be attributable to either of the alternatives considered. To what can the outer-line advantage be attributed? Attentional processes remain the most likely possibility. That is, inner and outer line elements may be equally visible, but subjects may employ an attentional strategy that gives priority to outer line components. This possibility gains some credibility when it is considered that in Experiments 1 and 2, in which a clear outer-line advantage was evident, subjects had to attend simultaneously to both the inner and outer areas of the form, whereas in Experiment 3, in which the effect was absent, subjects were forewarned about whether target lines were to be presented in inner or outer components of the form presented, and thus could attend selectively to the appropriate area of the form. Experiment 4 was designed to examine directly the degree to which an outer-line discriminative advantage depended upon attentional factors.

\section{EXPERIMENT 4}

There were two parts to Experiment 4. In one part a divided-attention condition was used, and in the other part a selective-attention condition was employed. In the selective-attention condition, target lines in inner and outer locations were presented on separate blocks, and subjects knew prior to presentation of a block whether inner or outer target locations were to be tested. In the dividedattention condition, inner and outer target lines were presented equally often in inner and outer locations, and subjects had no advance information about whether a target line would be in an inner or outer position. The assumption underlying the design was that if an attentionaltype strategy giving priority to outer-line targets was instrumental in determining the effect, then the two attentional conditions should produce very different results. 
In the divided-attention condition, the subjects were free to deploy attentional processes as they saw fit-thus, if an outer-line advantage results from subjects' giving attentional priority to outer line elements, the effect should be evident. In the selective-attention condition, the subjects were informed before a presentation block whether target lines would appear in the inner or outer areas. Under such circumstances, they should be able to allocate attention to the appropriate inner or outer locations and, as in Experiment 3, differences between inner and outer target lines should be minimal.

\section{Method}

Stimuli. The three forms shown in Panel B of Figure 1 were employed as stimuli. Individual forms were drawn in the same fashion as those employed in Experiment 1. The cross and octagon forms used were identical in size to those employed in Experiment 3 . The outer contour of the additional triangular form subtended a visual angle of $1.43^{\circ}$ in width and $1.29^{\circ}$ in height. The inner triangular form subtended a visual angle of $.76^{\circ}$.

Procedure and Subjects. In the divided-attention condition, each of the three forms was presented to a separate group of subjects. The subjects in all groups were provided with sample drawings of all versions of a given form, showing a thin line present in each different inner and outer position of the form. It was emphasized that a single thin line would be contained within a given form on each presentation of the form, and that a thin line would occur in the different locations, both inner and outer, equally often. The subjects were informed that their task was to discern the position of the thin line and then to point, on the response form provided, to the location at which the thin line was seen. The subjects were required to designate a location after each presentation, even if they were not entirely certain of the correctness of their response. Two practice blocks were used to familiarize subjects with the presentation procedure, followed by six blocks of experimental presentations. A thin line occurred equally often in all inner and outer positions within a block. Thirty-two-item presentation blocks were used for the octagon and cross forms, and 24-item presentation blocks for the triangular form. A 20-msec exposure duration was used throughout the experiment. The level of illumination of the exposure field was held fixed at $2.16 \mathrm{~cd} / \mathrm{m}^{2}$, and the level of illumination of the preexposure field was adjusted for each subject to ensure an overall performance level of $50 \%-80 \%$ correct responding. The average levels of illumination of the preexposure field were $2.31 \mathrm{~cd} / \mathrm{m}^{2}, 2.06 \mathrm{~cd} / \mathrm{m}^{2}$, and $2.74 \mathrm{~cd} / \mathrm{m}^{2}$ for the cross, octagon, and triangular forms, respectively. The 28 subjects were first-year undergraduates and participation was in exchange for course credit points. Twelve subjects were presented with the octagon form, and two separate groups of 8 subjects were presented with the cross and triangular forms.

In the selective-attention condition, the exposure duration and level of illumination of the exposure field were identical to those used in the divided-attention condition. The mean levels of illumination of the preexposure field (adjusted as before to ensure a performance level of $50 \%-80 \%$ correct responding for each subject) were $1.96 \mathrm{~cd} / \mathrm{m}^{2}, 2.88 \mathrm{~cd} / \mathrm{m}^{2}$, and $2.88 \mathrm{~cd} / \mathrm{m}^{2}$ for the octagon, cross, and triangular forms, respectively. The number and length of the presentation blocks were also identical to those used in the dividedattention condition. The conditions differed in that, in the dividedattention condition, presentation blocks containing inner target-line locations alternated with blocks containing outer target-line locations, and subjects were informed before each presentation block whether target lines would occur in the inner or outer areas of the form. The three different forms were presented to separate groups of 8 subjects each.

\section{Results}

Figure 4 shows the mean number of correct responses made to inner and outer target lines in the divided- and selective-attention conditions. A similar pattern is evident in the data obtained with all three different forms. In the divided-attention condition, in which no advance information was provided about the location of the target lines, subjects were, on the average, $14.3 \%$ more accurate in discerning outer line elements than in discerning inner line elements. In the selective-attention condition, in which subjects knew before each presentation whether the target line would be located in the inner or outer part of the form, there was a minimal $1.4 \%$ difference favoring outer line elements.

An analysis of variance was carried out on the number of correct responses made to inner and outer lines at each different spatial location in the forms utilized. Because the octagon and cross forms had target lines in the same position, the data obtained from these forms in both at-
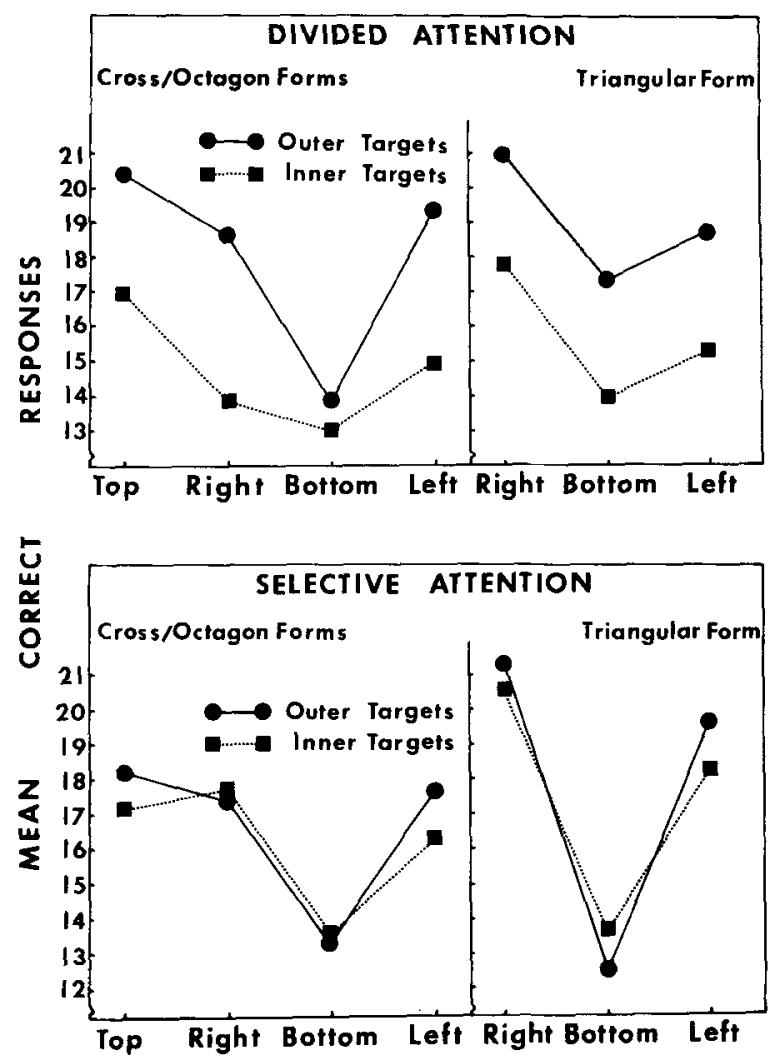

Figure 4. The upper part of the figure shows the mean number of correct responses made to inner and outer target lines at four different spatial locations under a divided-attention condition that required simultaneous attention to inner and outer lines. The lower part of the figure shows the mean number of correct responses made at the same locations under a selective-attention condition that required subjects to attend separately to inner and outer lines. The maximum number of correct responses possible in each condition was 24. 
tention conditions were examined in a single overall analysis. Data obtained in the two attention conditions with the triangular form were considered in an independent analysis. The cross/octagon analysis confirmed that outer lines were more accurately discerned than inner lines $[F(1,32)=13.95, p<.001]$, and that the outer-line advantage depended on whether subjects were in the dividedor selective-attention conditions $[F(1,32)=7.92, p<$ .01]. A similar pattern was evident in the analysis carried out on the triangular form. Outer line elements were more accurately discerned than inner line elements $[\mathrm{F}(1,14)=$ $13.91, p<.01]$, but again, this depended on whether subjects were in the divided- or selective-attention conditions $[F(1,14)=9.40, p<.01]$. Analyses performed only on the selective-attention conditions confirmed that there were no reliable differences in detection of targets at inner and outer locations in either the cross/octagon $[F(1,14)=1.05, p>.05]$ or the triangular form [F $<1]$. With the exception of spatial location of the target line, no other main effect or interaction approached significance.

Target lines were not discerned equally well at all spatial locations. A reliable position effect was evident in both the cross/octagon $[F(3,96)=13.96, p<.001]$ and the triangular form analyses $[\mathrm{F}(2,28)=13.48, \mathrm{p}<.001]$. As may be seen in Figure 4, the position effect primarily reflects subjects' poorer performance in responding to targets on the bottom parts of the forms used. A Scheffé test verified that the bottom position differed from the top, right, and left positions in the cross and octagon forms, and that the latter positions did not differ from one another $[t(96)=2.85, p<.05]$. Similarly, in the triangular form, the right and left sides of the triangle differed from the bottom, but not from each other $[\mathrm{t}(28)=2.59, \mathrm{p}<.05]$. A more detailed analysis of the position data suggests, as in Experiment 3, that the effect is largely attributable to a response bias. In responding, subjects designated the bottom locations of the forms much less frequently than other locations. This becomes quite apparent if we tabulate the frequency with which different responses were made on trials on which incorrect responses were given. For the cross/octagon forms, the mean number of erroneous responses made to the top, right, bottom, and left positions over a 24 -item presentation block were 10.33, 9.0, 4.78 , and 6.62 , respectively. For the triangular form, $9.72,4.22$, and 5.56 erroneous responses were made to the right, bottom, and left positions, respectively. A Scheffé test confirmed that fewer responses were made to the bottom position with both the cross/octagon forms $[F(3,96)=17.59, p<.05]$ and the triangular forms $[\mathrm{F}(2,28)=12.46, \mathrm{p}<.05]$.

\section{Discussion}

The results of Experiment 4 are straightforward. Targets in the outer lines of forms were discriminated more accurately than target lines in the inner areas. This finding held, however, only insofar as the subjects were required to attend simultaneously to inner and outer areas of the forms. Requiring subjects to attend to only the inner, or only the outer, line elements eliminated the outerline advantage. This pattern of data supports the view that an attentional strategy is the major determining factor generating the outer-line advantage in geometric forms.

\section{EXPERIMENT 5}

The preceding experiments confirmed that outer lines were more discriminable than inner lines in outline forms. In all of these experiments, however, the fixation point was located in the central area of the form. What needed to be determined was whether an outer-line advantage would be evident if the fixation point was positioned at a spatial location outside the form. Should an outer-line advantage not be evident under such conditions, it would impose substantial limitations on the generality of the effect, and would suggest that the outer-line advantage observed in the preceding experiments might be attributable to a retinally tied, peripheral-to-central scanning process. On the other hand, a finding of an outer-line advantage with an external fixation-point location would support the view that outside-in processing is a more general process that occurs independent of retinal location.

This issue has been raised with respect to the processing of letter strings. Merikle and his associates (e.g., Butler \& Merikle, 1973; Merikle, 1974) have assembled substantial evidence, using a backward masking paradigm, for a general "ends-in" scanning process. Others have provided support for a retinally tied, peripheral-to-central process (e.g., Mackworth, 1965; Schissler \& Baratta, 1972; White, 1976). No investigations have been carried out using more global geometric forms of the type used in the present series of experiments. Whether an outsidein effect will be evident with geometric stimuli and noncentral fixation-point locations remains an open issue.

\section{Method}

Stimuli and Design. A cross form, similar to that used in preceding studies, was employed as a stimulus. For half the subjects it was positioned above the fixation point, and for half the subjects, below the fixation point. Figure 5 shows the form and the arrangement used. The upper form was positioned so that half of the paired inner and outer target-line locations (lines $\mathrm{Bb}$ and $\mathrm{Cc}$ ) were equidistant from the fixation point. Similarly, the lower form was positioned so that half of the paired inner and outer target-line locations (lines $\mathrm{Aa}$ and $\mathrm{Dd}$ ) were equidistant from the fixation point. Thus, if an outer-line advantage reflects a retinally tied, peripheryto-fovea scan, differences would not be expected between these pairs of target lines, because they are equidistant from the fixation point. Differences would be expected between the other two pairs of inner and outer target lines, because the outer lines are more peripheral than inner lines. The rotated form subtended a visual angle of $1.3^{\circ}$ in both the horizontal and vertical directions.

Procedure and Subjects. The procedure was essentially similar to that used in the preceding experiments. The subjects were provided with sample drawings of the cross forms showing the target-line locations, and were required after each representation to point, on the response card shown in Figure 5, to the location at which a thin target line was evident. As in the preceding experiment, a single response line was used for a given pair of inner and outer lines. Thus, the subjects were not required to indicate whether a target line was located on an inner or outer part of the form, but only to designate its relative positions on the response form. One 


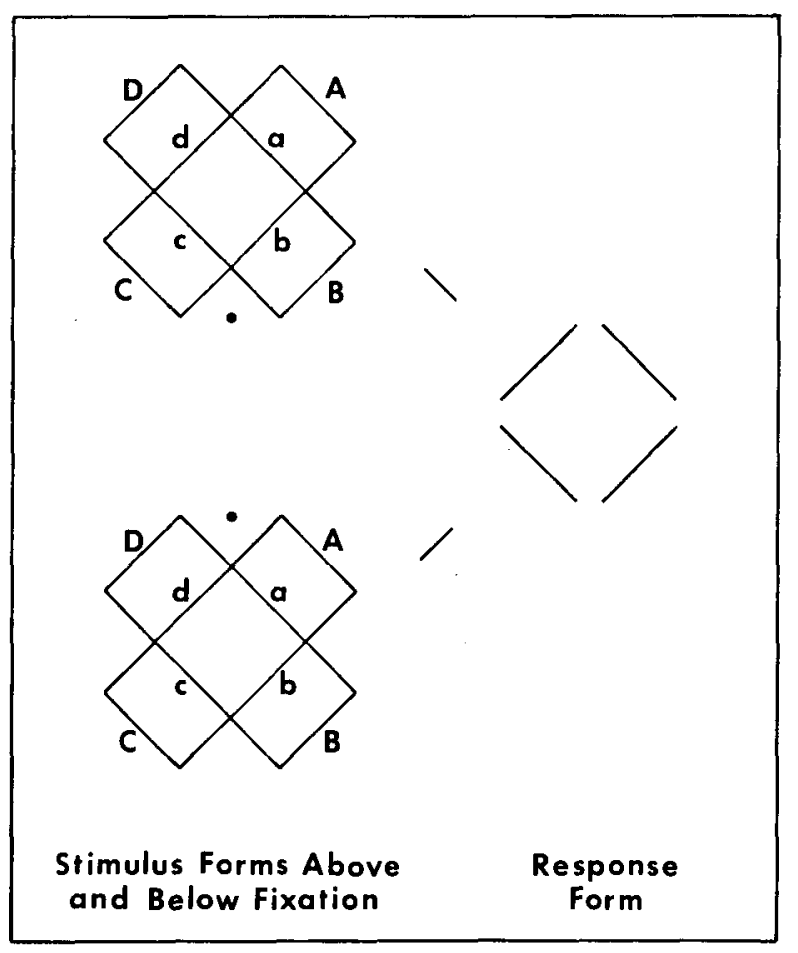

Figure 5. The rotated cross form used in Experiment 5 is shown located above the fixation point in the upper part of the figure, and below the fixation point in the lower part of the figure. The response form used by subjects to indicate their responses is shown on the right. Upper- and lowercase letters are used to indicate paired inner and outer target lines. In the upper form, inner and outer members of line pairs $\mathrm{Bb}$ and $\mathrm{Cc}$ are equidistant from fixation; in the lower form, inner and outer members of line pairs Aa and Dd are equidistant from fixation. Letters are included to clarify exposition only, and were not part of the stimulus presentation.

group of 8 subjects was presented with the form located above the fixation point, and another group of 8 subjects was presented with the form located below the fixation point. The 16 subjects were undergraduates who received course credit for participation. It was emphasized before each trial block that subjects were to activate the apparatus for a presentation only when their eyes were locked on the fixation-point location in the preexposure field. A $50-\mathrm{msec}$ exposure duration was used. The level of illumination of the exposure field was $2.86 \mathrm{~cd} / \mathrm{m}^{2}$ and the level of illumination of the preexposure field was adjusted for each subject to ensure an overall performance level of $50 \%-70 \%$ correct responding. The mean level of illumination of the preexposure field was $2.85 \mathrm{~cd} / \mathrm{m}^{2}$ when the fixation point was positioned above the form, and $2.30 \mathrm{~cd} / \mathrm{m}^{2}$ when the fixation point was positioned below the form. Six 32-item presentation blocks were preceded by two 32 -item practice blocks. The thin target line appeared equally often at all inner and outer locations within a presentation block.

\section{Results}

An analysis of variance was applied to the number of correct responses made to inner and outer line elements. As in the preceding experiments, requiring subjects to attend simultaneously to inner and outer line elements resulted in the more accurate discrimination of the outer line elements $[F(1,14)=30.10, p<.01]$. The difference favoring outer over inner line elements was $29.6 \%$ when the form was positioned above the fixation point and $14.1 \%$ when the form was positioned below the fixation point. This difference in the magnitude of the effect as a function of fixation-point location fell just short of significance $[F(9,141)=3.80, p<.05]$. The diminished magnitude of the outer-line effect with the form located below the fixation point may be related to the finding in the preceding experiment that subjects were less prone to direct attention to the lower parts of visual forms than to the upper parts of the visual field. Such a disposition may limit the effectiveness with which attentional strategies can be applied to stimulus configurations below the fixation point.

More interesting was the finding that the advantage of the outer-line targets was apparent when pairs of inner and outer lines were equidistant from the fixation point and when the outer target line was more peripheral than the inner line. The mean advantage of outer lines over inner lines when the inner and outer lines were equidistant from fixation was $19.1 \%$, and the mean advantage when the outer line was more peripheral than the inner line was $24.7 \%$. There was no indication of an interaction relating the size of the outer-line effect to the relative distance of inner and outer lines from the fixation point $[\mathrm{F}(1,14)=$ $1.50, p>.05]$. The only interaction involving the outerline advantage that proved reliable indicated a $12.3 \%$ larger outer-line advantage on the left than on the right side of the forms presented $[F(1,14)=7.62, p<.05]$. Why this should be the case is not clear. Inspection of the data shows no indication of an overall bias favoring responses to the left side of the form. Whether this larger advantage on the left represents a left-to-right scanning disposition occasioned by the rotation of the cross, such that the four arms fall half into the left and half into the right visual field, is open to speculation. The remaining reliable effect in the analysis served to confirm that inner and outer target-line locations more distant from the fixation point tended to be less accurately reported than target-line locations near the fixation point $[\mathrm{F}(1,14)=7.50$, $\mathrm{p}<.05$ ].

In summary, the data from this experiment are consonant with the findings of Experiment 4, which emphasized the role of voluntary attentional process in the processing of geometric forms. If voluntary attentional factors play a significant role in determining the outerline advantage, it follows that the location of the fixation point should not be a critical factor. The outer-line advantage should be evident whether the fixation point is located outside or inside the interior of the form.

\section{GENERAL DISCUSSION}

The objective of the experiments reported was to examine the discriminability of inner and outer line detail in geometric forms. Three findings merit comment. First, outer line elements were shown to be more accurately re- 
ported than inner line elements. This was apparent in Experiments 1, 4, and 5, which used the thin target-line discrimination task, and in Experiment 2, which used a more natural, holistic response and larger stimuli. Prior research has shown that, when alphabetic materials are presented, peripheral letters tend to be discriminated more accurately than inner letters (e.g., Mackworth, 1965), but such data has been lacking for structured geometric forms. The data show clearly that an outer-element advantage is apparent not only when isolated, random letter arrays are shown to the left and/or right of fixation, but also when global forms are presented, requiring concurrent registration of line structure above and below, as well as to the left and/or right of, fixation.

The second noteworthy finding was that attentional factors appear to play a major role in determining the outerline advantage that is evident with global forms. What makes this finding interesting is that it stands in marked contrast to the results of studies using letter arrays. Although exceptions exist (e.g., Wolford \& Chambers, 1983), there has been a general tendency in studies dealing with discriminability of letter elements to assign a very subordinate role to attentional processes. This is particularly evident in studies reported by Banks and his colleagues (e.g., Banks, Larson, \& Prinzmetal, 1979; Banks \& White, 1984), who have found that a peripheral letter advantage remains evident even when unlimited viewing time is allowed, even though such conditions should eliminate any need for the employment of viewing strategies. Banks and his colleagues accordingly downplay the importance of cognitive strategies in their explanation of greater discriminability of peripheral letters, and emphasize the role of sensory-based, retinally tied, Gestalt grouping processes. They propose that when a target letter and an adjacent mask are presented, they are perceived as a unitary Gestalt grouping. With the mask on the peripheral side of a given target letter located a specific distance from fixation, the grouped pair of elements will necessarily be further removed from fixation than would be the case if the mask were located on the central side of that same target letter. It follows that, because acuity falls off with distance from fixation, the more distant target and peripheral mask grouping will be recognized less accurately. It is assumed that such preattentive grouping mechanisms are responsible for observed effects, unless unusual presentation conditions are employed which call attentional mechanisms into play (see, e.g., Experiment 3 of Wolford \& Chambers, 1983). Banks and his colleagues have been able to marshal substantial amounts of evidence in support of this position. They have shown that differences in discriminatory accuracy associated with masking letters on the peripheral, as opposed to the central, side of target letters can be eliminated if a suitable correction is made for retinal eccentricity. They have also demonstrated that a peripheral-letter advantage remains evident even when the need for cognitive strategies is removed by using instructions and blocked-presentation sequences to eliminate uncertainty about target locations.
Although such a retinally tied acuity-gradient type of interpretation does serve to account for a good deal of experimentation with letter elements, it does not fare well when stimulus forms having a more global structure, such as those used in the present study, are presented. A blocked-presentation condition, coupled with information that target lines would occur either in inner or outer locations eliminated the outer-line advantage in Experiment 3. A more systematic examination of these factors in Experiment 4 confirmed that a strong outer-line advantage, evident when subjects were required to attend simultaneously to both the inner and outer components of three different forms, could be eliminated by blocking inner and outer target presentations of these forms and apprising the subject before each block as to whether target lines would be within or on the outside of the forms. In short, the data suggest that cognitive strategies play a much larger role with geometric forms than with letter arrays.

The third finding that merits comment was that the outer-line effect was evident both when the fixation point was outside and when it was within the geometric form presented. This finding is crucial to the acceptance of any general strategy-type interpretation is to be taken seriously. The outer-line effect should be largely independent of fixation-point location. The most prominent accounts developed to explain the greater discriminability of outer letters when letter arrays are presented dictate, on the other hand, that any outer-element advantage depends on fixation-point location. This holds with respect to either a retinally tied, peripheral-to-central scanning process such as that posited by White (1976), or an acuitygradient approach such as that advocated by Banks, Larson, and Prinzmetal (1979). Both accounts require outer line elements to be more peripheral if an outer-line advantage is to be evident. Contrary to this expectation, the data reported in Experiment 5 show a distinct outer-line advantage when the fixation point lay outside the form and inner and outer line elements were, on average, equidistant from fixation. Furthermore, in Experiments 1 and 4 , a robust outer-line effect was evident when inner and outer line elements exactly equidistant from the fixation point were compared. Once more, the data suggest that different mechanisms are at work in the processing of alphabetic and geometric materials.

Given that outer line elements are more accurately detected than inner line elements, and that attentional processes seem to be important in determining the difference, what remains to be discussed is why attention should be accorded to outer line elements when geometric forms were presented, and why attentional factors should be more evident with geometric stimuli than with alphabetic stimuli. As Ward (1983) has pointed out, finding that an aspect of a stimulus dominates information processing is one thing, and being able to specify the mechanism responsible for the effect is quite another. Our evidence suggests that a cognitive strategy is responsible for the outerline advantage, but the question of why attention should 
be focused on the outer line elements is not answered by any of the data provided in the experiments reported. A variety of possible reasons exist. One possibility is that outer line elements have temporal precedence, in the same way Navon (1977) suggests that global features are available before local features. Another possibility is that the outer line elements are perceptually more salient. A third possibility is that the outer-line advantage represents a developmental strategy. We are reluctant to accept either of the first two alternatives. With respect to temporal precedence, Ward (1983) has observed that this can be established only after a careful series of converging experiments. Such data are lacking. Furthermore, if outer line elements have temporal precedence, it is difficult to see why there should not have been an outer-line advantage even under blocked-presentation conditions. Temporal precedence should provide the same advantage whether inner and outer stimulus presentations are blocked or unblocked. As far as the second argument, that outer line elements are somehow perceptually more salient than inner lines, is concerned, the available evidence is not supportive. In Experiments 2 and 3, the discriminability of inner and outer target lines was assessed independently, and the outer-line advantage was eliminated. If the outer lines were somehow more salient, the effect should have remained evident under these conditions. It is the third possibility, that the outer-line effect is a developmental strategy, that appears most attractive. Certainly these results of Experiments 3, 4, and 5 strongly support the view that the outer-line advantage reflects the application of a developmental cognitive strategy. An attentional strategy favoring outer aspects of geometrical forms is evident early in childhood (Salapatek, 1975; Maurer, 1983), and its persistence into adulthood is to be expected. It is, after all, the outer line elements or edge structure that provide the most obvious source of information differentiating a given form from its background and other objects. As a form moves, or an individual moves with respect to a form, the major changes in the optical flow pattern occur at the outer edges of the form. What strategic advantage accrues to giving attentional priority to the outer boundaries of a form? The obvious possibility is that outer boundaries or discontinuities fix the expanse of the form and provide a basis for determining the direction and extent of eye movements that will facilitate the analysis of inner detail if the form is sufficiently large, or for determining the most efficient form of attentional shifts if it is not. Moreover, it is reasonable to expect that voluntary attentional strategies should be more pronounced when two- and three-dimensional geometric materials requiring multidirectional eye movements or attentional shifts are presented than when unidimensional letter strings allowing only bidirectional analytic operations are presented-as proved to be the case in the experiments reported here.

\section{REFERENCES}

BANKS, W. P., \& WHITE, H. (1984). Lateral interference and perceptual grouping in visual detection. Perception \& Psychophysics, 36, 285-295.

Banks, W. P., Larson, D. W., \& Prinzmetal, W. (1979). Asymmetry of visual interference. Perception \& Psychophysics, 25, 447-456.

BRYDEN, M. P. (1960). Tachistoscopic recognition of non-alphabetical material. Canadian Journal of Psychology, 14, 78-86.

BRYDEN, M. P., Dick, A. O., \& MEWHORT, J. K. (1968). Tachistoscopic recognition or number sequences. Canadian Journal of Psychology, 22, 52-59.

Butler, B., \& MerikLe, P. M. (1973). Selective masking and processing strategy. Quarterly Journal of Experimental Psychology, 25, 542-548.

EnNs, J. T., \& Prinzmetal, W. (1984). The role of redundancy in the object-line effect. Perception \& Psychophysics, 35, 22-32.

KinCHLA, R. A., \& WolfE, J. M. (1979). The order of visual processing: "Top-down," "bottom-up," or "tmiddle-out." Perception \& Psychophysics, 25, 225-231.

Lanze, M., Weisstein, N., \& Harris, J. R. (1982). Perceived depth vs. structural relevance in the object-superiority effect. Perception \& Psychophysics, 31, 376-382.

Mackworth, N. H. (1965). Visual noise causes tunnel vision. Psychonomic Science, 3, 67-68.

Maurer, D. (1983). The scanning of compound figures by young infants. Journal of Experimental Child Psychology, 35, 437-448.

McClelland, J. L., \& Miller, J. (1979). Structural factors in figure perception. Perception \& Psychophysics, 26, 221-229.

MeriKLe, P. M. (1974). Selective backward masking with an unpredictable mask. Journal of Experimental Psychology, 103, 589-591.

Navon, D. (1977). Forest before trees: The precedence of global features in visual perception. Cognitive Psychology, 9, 353-363.

Navon, D. (1981a). Do attention and decision follow perception? Comment on Miller. Journal of Experimental Psychology: Human Perception \& Performance, 7, 1175-1182.

Navon, D. (1981b). The forest revisited: More on global precedence. Psychological Research, 43, 1-32.

Navon, D., \& Norman, J. (1983). Does global precedence really depend on visual angle? Journal of Experimental Psychology: Human Perception \& Performance, 9, 955-965.

Pomerantz, J. R. (1983). Global and local precedence: Selective attention in form and motion perception. Joumal of Experimental Psychology: General, 112, 516-540.

Pomerantz, J. R., Sager, L. C., \& Stoever, R. J. (1977). Perception of wholes and of their component parts: Some configural superiority effects. Journal of Experimental Psychology: Human Perception \& Performance, 3, 422-435.

Prinzmetal, W., \& Banks, W. P. (1977). Good continuation affects visual detection. Perception \& Psychophysics, 21, 389-395.

Salapatek, P. (1975). Pattern perception in early infancy. In L. B. Cohen \& P. Salapatek (Eds.), Infant perception: From sensation to cognition: Vol. I. Basic visual processes. New York: Academic.

Schissler, D., \& Baratta, M. (1972). Evidence for an addition to the reading scan hypothesis. Perception \& Psychophysics, 12, 221-223.

Treisman, A., \& Paterson, R. (1984). Emergent features, attention, and object perception. Journal of Experimental Psychology: Human Perception \& Performance, 10, 12-31.

WALTZ, D. (1975). Understanding line drawings of scenes with shadows. In P. Winston (Ed.), The psychology of computer vision. New York: McGraw-Hill.

Ward, L. M. (1983). On processing dominance: Comment on Pomerantz. Journal of Experimental Psychology: General, 112, 541-546.

Weisstein, N., \& Harris, C. S. (1974). Visual detection of line segments: An object superiority effect. Science, 186, 752-755.

White, M. J. (1976). Order of processing in visual perception. Canadian Journal of Psychology, 30, 140-156.

Williams, M. C., \& Weisstein, N. (1978). Line segments are per- 
ceived better in a coherent context than alone: An object-line effect in visual perception. Memory \& Cognition, 6, 85-90.

Wolford, G., \& CHAMBers, L. (1983). Lateral masking as a function of spacing. Perception \& Psychophysics, 33, 129-138.

WOLFORD, G., \& HollingsworTh, S. (1974). Lateral masking in visual information processing. Perception \& Psychophysics, 16, 315-320.

\section{NOTE}

1. Studies of the object-superiority effect do examine the discriminability of single lines; however, they do this by using two or more phenomenally distinctive forms that differ with respect to the location of the line in question, and requiring subjects to determine which of the phenomenally distinctive forms was presented. Enns and Prinzmetal (1984) show that the object-line effect is probably attributable to the redundancies induced by phenomenological differences in the appearances of the forms generated by the different locations of the given line. According to this account, it is not the discrimination of a given line that is being examined in such experiments, but the distinctiveness in the overall character of the forms generated by the different positions of that line.

(Manuscript received August 21, 1984; revision accepted for publication September $2,1985$. ) 